\title{
Distance spectral radius of uniform hypergraphs
}

\author{
Hongying Lin, Bo Zhou ${ }^{\dagger}$ \\ School of Mathematical sciences, South China Normal University, \\ Guangzhou 510631, P. R. China
}

\begin{abstract}
We study the effect of three types of graft transformations to increase or decrease the distance spectral radius of uniform hypergraphs, and we determined the unique $k$-uniform hypertrees with maximum, second maximum, minimum and second minimum distance spectral radius, respectively.
\end{abstract}

AMS classification: $05 \mathrm{C} 50,05 \mathrm{C} 65,15 \mathrm{~A} 18$

Key words: distance spectral radius, uniform hypergraph, uniform hypertree, distance matrix, graft transformation

\section{Introduction}

A hypergraph $G$ consists of a vertex set $V(G)$ and an edge set $E(G)$, where $V(G)$ is nonempty, and each edge $e \in E(G)$ is a nonempty subset of $V(G)$, see [2]. The order of $G$ is $|V(G)|$. For an integer $k \geq 2$, we say that a hypergraph $G$ is $k$-uniform if every edge has size $k$. A (simple) graph is a 2-uniform hypergraph. The degree of a vertex $v$ in $G$, denoted by $d_{G}(v)$, is the number of edges of $G$ which contain $v$.

For $u, v \in V(G)$, a walk from $u$ to $v$ in $G$ is defined to be a sequence of vertices and edges $\left(v_{0}, e_{1}, v_{1}, \ldots, v_{p-1}, e_{p}, v_{p}\right)$ with $v_{0}=u$ and $v_{p}=v$ edge $e_{i}$ contains vertices $v_{i-1}$ and $v_{i}$, and $v_{i-1} \neq v_{i}$ for $i=1, \ldots, p$. The value $p$ is the length of this walk. A path is a walk with all $v_{i}$ distinct and all $e_{i}$ distinct. A cycle is a walk containing at least two edges, all $e_{i}$ are distinct and all $v_{i}$ are distinct except $v_{0}=v_{s}$. A vertex $u \in V(G)$ is viewed as a path (from $u$ to $u$ ) of length 0 . If there is a path from $u$ to $v$ for any $u, v \in V(G)$, then we say that $G$ is connected. A hypertree is a connected hypergraph with no cycles. Note that a $k$-uniform hypertree with $m$ edges always has order $1+(k-1) m$.

*E-mail: lhongying0908@126.com

${ }^{\dagger}$ Corresponding author. E-mail: zhoubo@scnu.edu.cn 
Let $G$ be a $k$-uniform hypergraph with $V(G)=\left\{v_{1}, \ldots, v_{n}\right\}$ and edge set $E(G)$, where $2 \leq k \leq n$. For $u, v \in V(G)$, the distance between $u$ and $v$ is the length of a shortest path from $u$ to $v$ in $G$, denoted by $d_{G}(u, v)$. In particular, $d_{G}(u, u)=0$. The diameter of $G$ is the maximum distance between all vertex pairs of $G$. The distance matrix of $G$ is the $n \times n$ matrix $D(G)=\left(d_{G}(u, v)\right)_{u, v \in V(G)}$. The eigenvalues of $D(G)$ are called the distance eigenvalues of $G$. Since $D(G)$ is real and symmetric, the distance eigenvalues of $G$ are real. The distance spectral radius of $G$, denoted by $\rho(G)$, is the largest distance eigenvalue of $G$. Note that $D(G)$ is a irreducible nonnegative matrix. The Perron-Frobenius theorem implies that $\rho(G)$ is simple, positive, and there is a unique positive unit eigenvector $x(G)$ corresponding to $\rho(G)$, which is called the distance Perron vector of $G$, denoted by $x(G)$.

The study of distance eigenvalues of 2-uniform hypergraphs (ordinary graphs) dates back to the classical work of Graham and Pollack [5], Graham and Lovász [4] and Edelberg et al. [3]. Ruzieh and Powers [7] showed that among connected 2-uniform hypergraphs of order $n$, the path $P_{n}$ is the unique graph with maximum distance spectral radius. Stevanović and Ilić [9] showed that among trees of order $n$, the star $S_{n}$ is the unique tree with minimum distance spectral radius. Nath and Paul [6] determined the unique trees with maximum distance spectral radius among trees with fixed matching number. For more details on distance eigenvalues and especially on distance spectral radius of 2-uniform hypergraphs, one may refer to the recent survey of Aouchiche and Hansen [1] and referees therein. Sivasubramanian [8] gave a formula for the inverse of a few $q$-analogs of the distance matrix of a 3-uniform hypertree.

For a $k$-uniform hypertree $G$ with $V(G)=\left\{v_{1}, \ldots, v_{n}\right\}$, if $E(G)=\left\{e_{1}, \ldots\right.$, $\left.e_{m}\right\}$, where $e_{i}=\left\{v_{(i-1)(k-1)+1}, \ldots, v_{(i-1)(k-1)+k}\right\}$ for $i=1, \ldots, m$, then we call $G$ a $k$-uniform loose path, denoted by $P_{n, k}$.

For a $k$-uniform hypertree $G$ of order $n$, if there is a disjoint partition of the vertex set $V(G)=\{u\} \cup V_{1} \cup \cdots \cup V_{m}$ such that $\left|V_{1}\right|=\cdots=\left|V_{m}\right|=k-1$, and $E(G)=\left\{\{u\} \cup V_{i}: 1 \leq i \leq m\right\}$, then we call $G$ is a hyperstar (with center $u$ ), denoted by $S_{n, k}$. In particular, $S_{1, k}$ is a hypergraph with a single vertex and $S_{k, k}$ is a hypergraph with a single edge.

In this paper, we study the effect of three types of graft transformations to increase or decrease the distance spectral radius of $k$-uniform hypergraphs. As applications, we show that $P_{n, k}$ and $S_{n, k}$ are the unique $k$-uniform hypertrees with maximum and minimum distance spectral radius, respectively, and we also determine the unique $k$-uniform hypertrees with second maximum and second minimum distance spectral radius, respectively.

\section{Preliminaries}

Let $G$ be a $k$-uniform hypergraph with $V(G)=\left\{v_{1}, \ldots, v_{n}\right\}$. A column vector $x=\left(x_{v_{1}}, \ldots, x_{v_{n}}\right)^{\top} \in \mathbb{R}^{n}$ can be considered as a function defined on $V(G)$ 
which maps vertex $v_{i}$ to $x_{v_{i}}$, i.e., $x\left(v_{i}\right)=x_{v_{i}}$ for $i=1, \ldots, n$. Then

$$
x^{\top} D(G) x=\sum_{\{u, v\} \subseteq V(G)} 2 d_{G}(u, v) x_{u} x_{v},
$$

and $\rho$ is a distance eigenvalue with corresponding eigenvector $x$ if and only if $x \neq 0$ and for each $u \in V(G)$,

$$
\rho x_{u}=\sum_{v \in V(G)} d_{G}(u, v) x_{v} .
$$

The above equation is called the $(\rho, x)$-eigenequation of $G$ (at $u)$. For a unit column vector $x \in \mathbb{R}^{n}$ with at least one nonnegative entry, by Rayleigh's principle, we have

$$
\rho(G) \geq x^{\top} D(G) x
$$

with equality if and only if $x=x(G)$.

For $u \in V(G)$, let $G-u$ be the sub-hypergraph of $G$ obtained by deleting $u$ and all edges containing $u$. We remark that in the literature this is sometimes denoted by strongly deleting the vertex $u$. For $e \in E(G)$, let $G-e$ be the sub-hypergraph of $G$ obtained by deleting $e$.

For $X \subseteq V(G)$ with $X \neq \emptyset$, let $G[X]$ be the sub-hypergraph induced by $X$, i.e., $G[X]$ has vertex set $X$ and edge set $\{e \cap X: e \in E(G)\}$, and let $\sigma_{G}(X)$ be the sum of the entries of the distance Perron vector of $G$ corresponding to the vertices in $X$.

Let $G$ be a $k$-uniform hypergraph with $u, v \in V(G)$ and $e_{1}, \ldots, e_{r} \in E(G)$ such that $u \notin e_{i}$ and $v \in e_{i}$ for $1 \leq i \leq r$. Let $e_{i}^{\prime}=\left(e_{i} \backslash\{v\}\right) \cup\{u\}$ for $1 \leq i \leq r$. Let $G^{\prime}$ be the hypergraph with $V\left(G^{\prime}\right)=V(G)$ and $E\left(G^{\prime}\right)=$ $\left(E(G) \backslash\left\{e_{1}, \ldots, e_{r}\right\}\right) \cup\left\{e_{1}^{\prime}, \ldots, e_{r}^{\prime}\right\}$. Then we say that $G^{\prime}$ is obtained from $G$ by moving edges $e_{1}, \ldots, e_{r}$ from $v$ to $u$.

A path $P=\left(v_{0}, e_{1}, v_{1}, \ldots, v_{p-1}, e_{p}, v_{p}\right)$ in a $k$-uniform hypergraph $G$ is called a pendant path at $v_{0}$, if $d_{G}\left(v_{0}\right) \geq 2, d_{G}\left(v_{i}\right)=2$ for $1 \leq i \leq p-1$, $d_{G}(v)=1$ for $v \in e_{i} \backslash\left\{v_{i-1}, v_{i}\right\}$ with $1 \leq i \leq p$, and $d_{G}\left(v_{p}\right)=1$. An edge $e=\left\{w_{1}, \ldots, w_{k}\right\}$ in $G$ is called a pendant edge at $w_{1}$ if $d_{G}\left(w_{1}\right) \geq 2, d_{G}\left(w_{i}\right)=1$ for $2 \leq i \leq k$.

If $P$ is a pendant path of a hypergraph $G$ at $u$, we say $G$ is obtained from $H$ by attaching a pendant path $P$ at $u$ with $H=G[V(G) \backslash(V(P) \backslash\{u\})]$. If $P$ is a pendant path of length one of $G$ at $u$, then we also say that $G$ is obtained from $H$ by attaching a pendant edge at $u$.

A component of a hypergraph $G$ is a maximal connected sub-hypergraph of $G$.

For vertex-disjoint hypergraphs $G_{1}$ and $G_{2}$, let $G_{1} \cup G_{2}$ the be union of $G_{1}$ and $G_{2}$, i.e, $V\left(G_{1} \cup G_{2}\right)=V\left(G_{1}\right) \cup V\left(G_{2}\right)$ and $E\left(G_{1} \cup G_{2}\right)=E\left(G_{1}\right) \cup E\left(G_{2}\right)$. 


\section{The effect of graft transformations on dis- tance spectral radius}

In this section we propose three types of graft transformations for a $k$-uniform hypergraph, and consider the effect of them to increase or decrease the distance spectral radius.

Let $G$ be a connected $k$-uniform hypergraph with $|E(G)| \geq 1$. For $u \in$ $V(G)$, and positive integers $p$ and $q$, let $G_{u}(p, q)$ be a $k$-uniform hypergraph obtained from $G$ by attaching two pendant paths of lengths $p$ and $q$ at $u$, respectively, and $G_{u}(p, 0)$ be a $k$-uniform hypergraph obtained from $G$ by attaching a pendant path of length $p$ at $u$.

Theorem 3.1. Let $G$ be a connected $k$-uniform hypergraph $|E(G)| \geq 1$ and $u \in V(G)$. For integers $p \geq q \geq 1, \rho\left(G_{u}(p, q)\right)<\rho\left(G_{u}(p+1, q-1)\right)$.

Proof. Let $H=G_{u}(p, q)$. Let $P=\left(u, e_{1}, u_{1}, \ldots, u_{p-1}, e_{p}, u_{p}\right)$ and $Q=\left(u, e_{1}^{\prime}, v_{1}\right.$, $\left.\ldots, v_{q-1}, e_{q}^{\prime}, v_{q}\right)$ be the pendant paths of $H$ at $u$ of lengths $p$ and $q$, respectively. Let $e_{u}^{1}, \ldots, e_{u}^{t}$ be all the edges of $G$ containing $u$, where $t \geq 1$.

Suppose that $\sigma_{H}(V(P)) \geq \sigma_{H}(V(Q))$. Let $H^{\prime}$ be the hypergraph obtained from $H$ by moving edges $e_{u}^{1}, \ldots, e_{u}^{t}$ from $u$ to $v_{1}$. It is easily seen that $H^{\prime} \cong$ $G_{u}(p+1, q-1)$. As we pass from $H$ to $H^{\prime}$, the distance between a vertex of $V(G) \backslash\{u\}$ and a vertex of $V(P)$ is increased by 1 , the distance between a vertex of $V(G) \backslash\{u\}$ and $V(Q) \backslash\left(e_{1}^{\prime} \backslash\left\{v_{1}\right\}\right)$ is decreased by 1, and the distance between any other vertex pair remains unchanged. Let $x=x(H)$. Then

$$
\begin{aligned}
& \frac{1}{2}\left(\rho\left(H^{\prime}\right)-\rho(H)\right) \\
\geq & \frac{1}{2} x^{\top}\left(D\left(H^{\prime}\right)-D(H)\right) x \\
= & \sigma_{H}(V(G) \backslash\{u\})\left[\sigma_{H}(V(P))-\left(\sigma_{H}(V(Q))-\sigma_{H}\left(e_{1}^{\prime} \backslash\left\{v_{1}\right\}\right)\right)\right] \\
= & \sigma_{H}(V(G) \backslash\{u\})\left[\sigma_{H}(V(P))-\sigma_{H}(V(Q))+\sigma_{H}\left(e_{1}^{\prime} \backslash\left\{v_{1}\right\}\right)\right] \\
\geq & \sigma_{H}(V(G) \backslash\{u\}) \sigma_{H}\left(e_{1}^{\prime} \backslash\left\{v_{1}\right\}\right) \\
> & 0
\end{aligned}
$$

and thus $\rho(H)<\rho\left(H^{\prime}\right)$.

Suppose that $\sigma_{H}(V(P))<\sigma_{H}(V(Q))$. Let $H^{\prime \prime}$ be the hypergraph obtained from $H$ by moving edges $e_{u}^{1}, \ldots, e_{u}^{t}$ from $u$ to $u_{1}$. It is easily seen that $H^{\prime \prime} \cong$ $G_{u}(p-1, q+1)$. By similar argument as above, we have that $\rho(H)<\rho\left(H^{\prime \prime}\right)$.

Thus we have proved that

$$
\rho\left(G_{u}(p, q)\right)<\max \left\{\rho\left(G_{u}(p-1, q+1)\right), \rho\left(G_{u}(p+1, q-1)\right)\right\} .
$$

If $p=q$, then the result follows. Suppose that $p>q$. If $\rho\left(G_{u}(p, q)\right)<$ $\rho\left(G_{u}(p-1, q+1)\right)$, then by using (3.1) repeatedly, we have

$$
\rho\left(G_{u}(p, q)\right) \leq \rho\left(G_{u}\left(\left\lceil\frac{p+q+1}{2}\right\rceil,\left\lfloor\frac{p+q-1}{2}\right\rfloor\right)\right)
$$




$$
<\rho\left(G_{u}\left(\left\lfloor\frac{p+q-1}{2}\right\rfloor,\left\lceil\frac{p+q+1}{2}\right\rceil\right)\right),
$$

which is impossible. Thus $\rho\left(G_{u}(p, q)\right)<\rho\left(G_{u}(p+1, q-1)\right)$.

Let $G$ be a connected $k$-uniform hypergraph with $u, v \in e \in E(G)$. For positive integers $p$ and $q$, let $G_{u, v}(p, q)$ be a $k$-uniform hypergraph obtained from $G$ by attaching a pendant path of length $p$ at $u$ and a pendant path of length $q$ at $v$, and $G_{u, v}(p, 0)$ be a $k$-uniform hypergraph obtained from $G$ by attaching a pendant path of length $p$ at $u$. Let $G_{u, v}(0, q)=G_{v, u}(q, 0)$.

Theorem 3.2. Let $G$ be a connected $k$-uniform hypergraph $|E(G)| \geq 2$ and $u, v \in e \in E(G)$. Suppose that $G-e$ consists of $k$ components. For integers $p, q \geq 1, \rho\left(G_{u, v}(p, q)\right)<\rho\left(G_{u, v}(p-1, q+1)\right)$ or $\rho\left(G_{u, v}(p, q)\right)<\rho\left(G_{u, v}(p+\right.$ $1, q-1))$.

Proof. Let $H=G_{u, v}(p, q)$. Let $P=\left(u, e_{1}, u_{1}, \ldots, u_{p-1}, e_{p}, u_{p}\right)$ and $Q=$ $\left(v, e_{1}^{\prime}, v_{1}, \ldots, v_{q-1}, e_{q}^{\prime}, v_{q}\right)$ be the pendant paths of $H$ at $u$ and $v$ of lengths $p$ and $q$, respectively. Let $e=\left\{u, v, w_{1}, \ldots, w_{k-2}\right\}, e_{1}=\left\{u, u_{1}, w_{1}^{\prime}, \ldots, w_{k-2}^{\prime}\right\}$ and $e_{1}^{\prime}=\left\{v, v_{1}, w_{1}^{\prime \prime}, \ldots, w_{k-2}^{\prime \prime}\right\}$. For $w \in e$, let $G_{w}$ be the component of $G-e$ containing $w$, and let $t_{w}$ be the number of edges of $G-e$ containing $w$. For $w \in e$ with $t_{w} \geq 1$, let $e_{w}^{1}, \ldots, e_{w}^{t_{w}}$ be the $t_{w}$ edges of $G-e$ containing $w$. Note that $e_{w}^{1}, \ldots, e_{w}^{t_{w}} \in E\left(G_{w}\right)$ for $w \in e$.

Suppose that $\sigma_{H}(V(P)) \geq \sigma_{H}(V(Q))$. Let $H^{\prime}$ be the hypergraph obtained from $H$ by moving edges $e_{w_{i}}^{1}, \ldots, e_{w_{i}}^{t_{w_{i}}}$ from $w_{i}$ to $w_{i}^{\prime \prime}$ for all $i$ with $1 \leq i \leq$ $k-2$ and $t_{i} \geq 1$ if $k \geq 3$, moving edges $e_{u}^{1}, \ldots, e_{u}^{t_{u}}$ from $u$ to $v$ if $t_{u} \geq 1$, and moving edges $e_{v}^{1}, \ldots, e_{v}^{t_{v}}$ from $v$ to $v_{1}$ if $t_{v} \geq 1$. It is easily seen that $H^{\prime} \cong G_{u, v}(p+1, q-1)$.

For $k \geq 3$, let $I=\left\{i: 1 \leq i \leq k-2, t_{w_{i}} \geq 1\right\}$. As we pass from $H$ to $H^{\prime}$, the distance between a vertex of $V\left(G_{u}\right) \backslash\{u\}$ and a vertex of $V(P)$ is increased by 1 , and the distance between a vertex of $V\left(G_{u}\right) \backslash\{u\}$ and a vertex of $V(Q)$ is decreased by 1 , the distance between a vertex of $V\left(G_{v}\right) \backslash\{v\}$ and a vertex of $V(P) \cup(e \backslash\{u\})$ is increased by 1, the distance between a vertex of $V\left(G_{v}\right) \backslash\{v\}$ and a vertex of $V(Q) \backslash\left(e_{1}^{\prime} \backslash\left\{v_{1}\right\}\right)$ is decreased by 1 , for $i \in I$ with $k \geq 3$, the distance between a vertex of $V\left(G_{w_{i}}\right) \backslash\left\{w_{i}\right\}$ and a vertex of $V(P) \cup\left(e \backslash\left\{w_{i}, u, v\right\}\right)$ is increased by 1 , the distance between a vertex of $V\left(G_{w_{i}}\right) \backslash\left\{w_{i}\right\}$ and $w_{i}$ is increased by 2 , the distance between a vertex of $V\left(G_{w_{i}}\right) \backslash\left\{w_{i}\right\}$ and a vertex of $V(Q) \backslash\left\{v, w_{i}^{\prime \prime}\right\}$ is decreased by 1 , and the distance between a vertex of $V\left(G_{w_{i}}\right) \backslash\left\{w_{i}\right\}$ and $w_{i}^{\prime \prime}$ is decreased by 2 , and the distance between any other vertex pair remains unchanged. Let $x=x(H)$. Note that $\sigma_{H}(e)-x_{u} \geq x_{v}$ and $\sigma_{H}\left(e_{1}^{\prime}\right)-x_{v_{1}} \geq x_{v}$. Then

$$
\begin{aligned}
& \frac{1}{2}\left(\rho\left(H^{\prime}\right)-\rho(H)\right) \\
\geq & \frac{1}{2} x^{\top}\left(D\left(H^{\prime}\right)-D(H)\right) x \\
= & \sigma_{H}\left(V\left(G_{u}\right) \backslash\{u\}\right)\left(\sigma_{H}(V(P))-\sigma_{H}(V(Q))\right)
\end{aligned}
$$




$$
\begin{aligned}
& +\sigma_{H}\left(V\left(G_{v}\right) \backslash\{v\}\right)\left[\sigma_{H}(V(P))+\left(\sigma_{H}(e)-x_{u}\right)\right. \\
& \left.-\left(\sigma_{H}(V(Q))-\left(\sigma_{H}\left(e_{1}^{\prime}\right)-x_{v_{1}}\right)\right)\right] \\
& +\sum_{i \in I} \sigma_{H}\left(V\left(G_{w_{i}}\right) \backslash\left\{w_{i}\right\}\right)\left[\sigma_{H}(V(P))+\left(\sigma_{H}(e)-x_{w_{i}}-x_{u}-x_{v}\right)+2 x_{w_{i}}\right. \\
& \left.-\left(\sigma_{H}(V(Q))-x_{v}-x_{w_{i}^{\prime \prime}}\right)-2 x_{w_{i}^{\prime \prime}}\right] \\
= & \sigma_{H}\left(V\left(G_{u}\right) \backslash\{u\}\right)\left(\sigma_{H}(V(P))-\sigma_{H}(V(Q))\right) \\
& +\sigma_{H}\left(V\left(G_{v}\right) \backslash\{v\}\right)\left[\sigma_{H}(V(P))-\sigma_{H}(V(Q))\right. \\
& \left.+\left(\sigma_{H}(e)-x_{u}\right)+\left(\sigma_{H}\left(e_{1}^{\prime}\right)-x_{v_{1}}\right)\right] \\
& +\sum_{i \in I} \sigma_{H}\left(V\left(G_{w_{i}}\right) \backslash\left\{w_{i}\right\}\right)\left[\sigma_{H}(V(P))-\sigma_{H}(V(Q))\right. \\
& \left.+\left(\sigma_{H}(e)-x_{u}\right)+x_{w_{i}}-x_{w_{i}^{\prime \prime}}\right] \\
\geq & \sigma_{H}\left(V\left(G_{u}\right) \backslash\{u\}\right)\left(\sigma_{H}(V(P))-\sigma_{H}(V(Q))\right) \\
& +\sigma_{H}\left(V\left(G_{v}\right) \backslash\{v\}\right)\left(\sigma_{H}(V(P))-\sigma_{H}(V(Q))+2 x_{v}\right) \\
& +\sum_{i \in I} \sigma_{H}\left(V\left(G_{w_{i}}\right) \backslash\left\{w_{i}\right\}\right)\left(\sigma_{H}(V(P))-\sigma_{H}(V(Q))+x_{v}+x_{w_{i}}-x_{w_{i}^{\prime \prime}}\right) \\
\geq & 2 \sigma_{H}\left(V\left(G_{v}\right) \backslash\{v\}\right) x_{v}+\sum_{i \in I} \sigma_{H}\left(V\left(G_{w_{i}}\right) \backslash\left\{w_{i}\right\}\right)\left(x_{v}+x_{w_{i}}-x_{w_{i}^{\prime \prime}}\right)
\end{aligned}
$$

By the eigenequations of $H$ at $v, w_{i}$ and $w_{i}^{\prime \prime}$, we have

$$
\begin{aligned}
\rho(H) x_{v} & =x_{w_{i}}+x_{w_{i}^{\prime \prime}}+\sum_{w \in V(H) \backslash\left\{v, w_{i}, w_{i}^{\prime \prime}\right\}} d_{H}(v, w), \\
\rho(H) x_{w_{i}} & =x_{v}+2 x_{w_{i}^{\prime \prime}}+\sum_{w \in V(H) \backslash\left\{v, w_{i}, w_{i}^{\prime \prime}\right\}} d_{H}\left(w_{i}, w\right), \\
\rho(H) x_{w_{i}^{\prime \prime}} & =2 x_{w_{i}}+x_{v}+\sum_{w \in V(H) \backslash\left\{v, w_{i}, w_{i}^{\prime \prime}\right\}} d_{H}\left(w_{i}^{\prime \prime}, w\right) .
\end{aligned}
$$

For $w \in V(H) \backslash\left\{v, w_{i}, w_{i}^{\prime \prime}\right\}$ with $i \in I$, we have $d_{H}(v, w)+d_{H}\left(w_{i}, w\right)-$ $d_{H}\left(w_{i}^{\prime \prime}, w\right) \geq 0$. Then $\rho(H)\left(x_{v}+x_{w_{i}}-x_{w_{i}^{\prime \prime}}\right) \geq-x_{w_{i}}+3 x_{w_{i}^{\prime \prime}}$. Since $(\rho(H)+$ 1) $\left(x_{v}+x_{w_{i}}-x_{w_{i}^{\prime \prime}}\right) \geq x_{v}+2 x_{w_{i}^{\prime \prime}}>0$, we have $x_{v}+x_{w_{i}}-x_{w_{i}^{\prime \prime}}>0$. Thus

$$
\begin{aligned}
\frac{1}{2}\left(\rho\left(H^{\prime}\right)-\rho(H)\right) \geq & \frac{1}{2} x^{\top}\left(D\left(H^{\prime}\right)-D(H)\right) x \\
\geq & 2 \sigma_{H}\left(V\left(G_{v}\right) \backslash\{v\}\right) x_{v} \\
& +\sum_{i \in I} \sigma_{H}\left(V\left(G_{w_{i}}\right) \backslash\left\{w_{i}\right\}\right)\left(x_{v}+x_{w_{i}}-x_{w_{i}^{\prime \prime}}\right) \\
\geq & 0 .
\end{aligned}
$$

It follows that $\rho(H) \leq \rho\left(H^{\prime}\right)$.

If $I \neq \emptyset$, then $\sum_{i \in I} \sigma_{H}\left(V\left(G_{w_{i}}\right) \backslash\left\{w_{i}\right\}\right)>0$, and thus from (3.2) we have $\rho(H)<\rho\left(H^{\prime}\right)$. Suppose that $I=\emptyset$ and $\rho(H)=\rho\left(H^{\prime}\right)$. Then $\sigma_{H}\left(V\left(G_{v}\right) \backslash\right.$ $\{v\})=0$, i.e., $V\left(G_{v}\right)=\{v\}$. Since $|E(G)| \geq 2$, we have $V\left(G_{u}\right) \backslash\{u\} \neq$ $\emptyset$. Thus $d_{H}(u, z)<d_{H^{\prime}}(u, z)$ for $z \in V\left(G_{u}\right) \backslash\{u\}$. On the other hand, 
since all inequalities in (3.2) are equalities, we have $x^{\top} D\left(H^{\prime}\right) x=\rho\left(H^{\prime}\right)$, and thus $x\left(H^{\prime}\right)=x$, implying that $\rho(H) x_{u}=(D(H) x)_{u}<\left(D\left(H^{\prime}\right) x\right)_{u}=\rho\left(H^{\prime}\right) x_{u}$ contradiction. Thus $\rho(H)<\rho\left(H^{\prime}\right)$.

Suppose that $\sigma_{H}(V(P))<\sigma_{H}(V(Q))$. Let $H^{\prime \prime}$ be the hypergraph obtained from $H$ by moving edges $e_{w_{i}}^{1}, \ldots, e_{w_{i}}^{t_{w_{i}}}$ from $w_{i}$ to $w_{i}^{\prime}$ for all $i$ with $1 \leq i \leq k-2$ and $t_{i} \geq 1$ if $k \geq 3$, moving edges $e_{u}^{1}, \ldots, e_{u}^{t_{u}}$ from $v$ to $u$ if $t_{u} \geq 1$, and moving edges $e_{v}^{1}, \ldots, e_{v}^{t_{v}}$ from $u$ to $u_{1}$ if $t_{v} \geq 1$. It is easily seen that $H^{\prime \prime} \cong$ $G_{u, v}(p-1, q+1)$. By similar argument as above, we have that $\rho(H)<\rho\left(H^{\prime \prime}\right)$.

Thus $\rho\left(G_{u, v}(p, q)\right)<\rho\left(G_{u, v}(p-1, q+1)\right)$ or $\rho\left(G_{u, v}(p, q)\right)<\rho\left(G_{u, v}(p+1, q-\right.$ $1))$, as desired.

Corollary 3.1. Let $G$ be a connected $k$-uniform hypergraph with $|E(G)| \geq 2$ and $u, v \in e \in E(G)$. Suppose that $G-e$ consists of $k$ components and $d_{G}(u)=d_{G}(v)=1$. For $p \geq q \geq 1, \rho\left(G_{u, v}(p, q)\right)<\rho\left(G_{u, v}(p+1, q-1)\right)$.

Proof. If $p=q$, then the result follows from Theorem 3.2. Suppose that $p>q$. Suppose that $\rho\left(G_{u, v}(p, q)\right)<\rho\left(G_{u, v}(p-1, q+1)\right)$. Since $d_{G}(u)=$ $d_{G}(v)=1$, we have $G_{u, v}\left(\left\lceil\frac{p+q+1}{2}\right\rceil,\left\lfloor\frac{p+q-1}{2}\right\rfloor\right) \cong G_{u, v}\left(\left\lfloor\frac{p+q-1}{2}\right\rfloor,\left\lceil\frac{p+q+1}{2}\right\rceil\right)$. Using Theorem 3.2 repeatedly, we have $\rho\left(G_{u, v}(p, q)\right) \leq \rho\left(G_{u, v}\left(\left\lceil\frac{p+q+1}{2}\right\rceil,\left\lfloor\frac{p+q-1}{2}\right\rfloor\right)\right)<$ $\rho\left(G_{u, v}\left(\left\lfloor\frac{p+q-1}{2}\right\rfloor,\left\lceil\frac{p+q+1}{2}\right\rceil\right)\right)$, which is impossible. Thus $\rho\left(G_{u, v}(p, q)\right)<\rho\left(G_{u, v}(p+\right.$ $1, q-1))$.

Let $G$ be a connected $k$-uniform hypergraph with $|E(G)| \geq 2$, and let $e=\left\{w_{1}, \ldots, w_{k}\right\}$ be an edge of $G$, where $d_{G}\left(w_{i}\right)=1$ for $1 \leq i \leq k-1$, $d_{G}\left(w_{k}\right) \geq 2$. For $1 \leq i \leq k-1$, let $H_{i}$ be a connected $k$-uniform hypergraph with $v_{i} \in V\left(H_{i}\right)$. Suppose that $G, H_{1}, \ldots, H_{k-1}$ are vertex-disjoint. For $0 \leq$ $s \leq k-1$, let $G_{e, s}\left(H_{1}, \ldots, H_{k-1}\right)$ be the hypergraph obtained by identifying $w_{i}$ of $G$ and $v_{i}$ of $H_{i}$ for $s+1 \leq i \leq k-1$ and identifying $w_{k}$ of $G$ and $v_{i}$ of $H_{i}$ for all $i$ with $1 \leq i \leq s$. In particular, if $H_{i}=S_{t_{i}(k-1)+1, k}$ and $v_{i}$ is its heart, where $t_{i} \geq 0$ and $1 \leq i \leq k-1$, then we write $G_{e, s}\left(t_{1}, \ldots, t_{k-1}\right)$ for $G_{e, s}\left(H_{1}, \ldots, H_{k-1}\right)$.

Theorem 3.3. Suppose that $\left|E\left(H_{j}\right)\right| \geq 1$ for some $j$ with $1 \leq j \leq k-1$. Then $\rho\left(G_{e, 0}\left(H_{1}, \ldots, H_{k-1}\right)\right)>\rho\left(G_{e, s}\left(H_{1}, \ldots, H_{k-1}\right)\right)$ for $j \leq s \leq k-1$.

Proof. Let $H=G_{e, 0}\left(H_{1}, \ldots, H_{k-1}\right)$. For $1 \leq i \leq k-1$, let $t_{i}$ be the number of edges of $H-e$ containing $w_{i}$. For $1 \leq i \leq k-1$ with $t_{i} \geq 1$, let $e_{i}^{1}, \ldots, e_{i}^{t_{i}}$ be the $t_{i}$ edges of $H-e$ containing $w_{i}$. Let $H^{\prime}$ be the hypergraph obtained by moving edges $e_{i}^{1}, \ldots, e_{i}^{t_{i}}$ from $w_{i}$ to $w_{k}$ for all $i$ with $1 \leq i \leq s$ and $t_{i} \geq 1$. It is easily seen that $H^{\prime} \cong G_{e, s}\left(H_{1}, \ldots, H_{k-1}\right)$.

Let $I=\left\{i: 1 \leq i \leq s, t_{i} \geq 1\right\}$. As we pass from $H$ to $H^{\prime}$, for $i, l \in I$ with $i \neq l$, the distance between a vertex of $V\left(H_{i}\right) \backslash\left\{w_{i}\right\}$ and a vertex of $V\left(H_{l}\right) \backslash\left\{w_{l}\right\}$ is decreased by 1 , the distance between a vertex of $V\left(H_{i}\right) \backslash\left\{w_{i}\right\}$ and a vertex of $(V(G) \backslash e) \cup\left\{w_{k}\right\}$ is decreased by 1 , the distance between a vertex of $V\left(H_{i}\right) \backslash\left\{w_{i}\right\}$ and $w_{i}$ is increased by 1 , and the distance between any 
other vertex pair remains unchanged. Let $x=x\left(H^{\prime}\right)$. Then

$$
\begin{aligned}
& \frac{1}{2}\left(\rho(H)-\rho\left(H^{\prime}\right)\right) \\
\geq & \frac{1}{2} x^{\top}\left(D(H)-D\left(H^{\prime}\right)\right) x \\
= & \sum_{i \in I} \sigma_{H^{\prime}}\left(V\left(H_{i}\right) \backslash\left\{w_{i}\right\}\right)\left(\sum_{l \in I} \sigma_{H^{\prime}}\left(V\left(H_{l}\right) \backslash\left\{w_{l}\right\}\right)\right. \\
& \left.+\sigma_{H^{\prime}}(V(G) \backslash e)+x_{w_{k}}-x_{w_{i}}\right) \\
\geq & \sum_{i \in I} \sigma_{H^{\prime}}\left(V\left(H_{i}\right) \backslash\left\{w_{i}\right\}\right)\left(\sigma_{H^{\prime}}(V(G) \backslash e)+x_{w_{k}}-x_{w_{i}}\right) \\
\geq & \sigma_{H^{\prime}}\left(V\left(H_{t}\right) \backslash\left\{w_{j}\right\}\right)\left(\sigma_{H^{\prime}}(V(G) \backslash e)+x_{w_{k}}-x_{w_{j}}\right) .
\end{aligned}
$$

Note that there is an edge different from $e$ containing $w_{k}$. Let $v$ be a vertex different from $w_{k}$ in such an edge of $G$. For $w \in V(H) \backslash\left\{v, w_{k}, w_{j}\right\}$, we have $d_{H^{\prime}}(v, w)+d_{H^{\prime}}\left(w_{k}, w\right)-d_{H^{\prime}}\left(w_{j}, w\right) \geq 0$. By the eigenequations of $H^{\prime}$ at $v, w_{k}$ and $w_{j}$, we have $\rho\left(H^{\prime}\right)\left(x_{v}+x_{w_{k}}-x_{w_{j}}\right) \geq-x_{v}+3 x_{w_{j}}$. Since $\left(\rho\left(H^{\prime}\right)+\right.$ 1) $\left(x_{v}+x_{w_{k}}-x_{w_{j}}\right) \geq x_{w_{k}}+2 x_{w_{j}}>0$, we have $x_{v}+x_{w_{k}}-x_{w_{j}}>0$. Then $\sigma_{H^{\prime}}(V(G) \backslash e)+x_{w_{k}}-x_{w_{j}}>x_{v}+x_{w_{k}}-x_{w_{j}}>0$. From (3.3), we have $\rho(H)>\rho\left(H^{\prime}\right)$.

Corollary 3.2. For $G_{e, 0}\left(t_{1}, \ldots, t_{k-1}\right)$, if $t_{j} \geq 1$ for some $j$ with $1 \leq j \leq k-1$, then $\rho\left(G_{e, 0}\left(t_{1}, \ldots, t_{k-1}\right)\right)>\rho\left(G_{e, s}\left(t_{1}, \ldots, t_{k-1}\right)\right.$ for $j \leq s \leq k-1$.

\section{Distance spectral radius of uniform hyper- trees}

In this section we study the distance spectral radius of $k$-uniform hypertrees using the results in Section 3.

For positive integers $\Delta, n$ with $1 \leq \Delta \leq \frac{n-1}{k-1}$, let $B_{n, k}^{\Delta}$ be the $k$-uniform hypertree obtained from vertex-disjoint hyperstar $S_{(\Delta-1)(k-1)+1, k}$ with center $u$ and loose path $P_{n-(\Delta-1)(k-1), k}$ with an end vertex $v$ by identifying $u$ and $v$. In particular, $B_{n, k}^{\Delta} \cong P_{n, k}$ if $\Delta=1,2$.

Theorem 4.1. Let $T$ be a $k$-uniform hypertree on $n$ vertices with maximum degree $\Delta$, where $1 \leq \Delta \leq \frac{n-1}{k-1}$. Then $\rho(T) \leq \rho\left(B_{n, k}^{\Delta}\right)$ with equality if and only if $T \cong B_{n, k}^{\Delta}$.

Proof. It is trivial if $\Delta=1$. Suppose that $\Delta \geq 2$. Let $T$ be a $k$-uniform hypertree of order $n$ with maximum degree $\Delta$ having maximum distance spectral radius.

Let $u$ be a vertex of $T$ with degree $\Delta$.

Case 1. $\Delta \geq 3$.

Suppose that there are at least two vertices of degree at least 3 in $T$. Choose a vertex $v$ of degree at least 3 such that $d_{T}(u, v)$ is as large as possible. Let $T_{1}, \ldots, T_{d_{T}(v)}$ be the vertex disjoint sub-hypergraphs of $T-v$ such that $T\left[V\left(T_{i}\right) \cup\{v\}\right]$ is a $k$-uniform hypertree for $1 \leq i \leq d_{T}(v)$. Assume that 
$u \in V\left(T_{1}\right)$. If $k=2$, then $T\left[V\left(T_{i}\right) \cup\{v\}\right]$ is a pendant path at $v$ for $2 \leq$ $i \leq d_{T}(v)$. Suppose that $k \geq 3$ and $T\left[V\left(T_{i}\right) \cup\{v\}\right]$ is not a pendant path at $v$ for some $i$ with $2 \leq i \leq d_{T}(v)$. Then there is at least one edge in $E\left(T\left[V\left(T_{i}\right) \cup\{v\}\right]\right)$ with at least three vertices of degree two. We choose such an edge $e=\left\{w_{1}, \ldots, w_{k}\right\}$ by requiring that $d_{T}\left(v, w_{1}\right)$ is as large as possible, where $d_{T}\left(v, w_{1}\right)=d_{T}\left(v, w_{j}\right)-1$ for $2 \leq j \leq k$. Then there are at least two pendant paths at different vertices of $e$, say $P=\left(w_{j}, e_{1}, u_{1}, \ldots, u_{p-1}, e_{p}, u_{p}\right)$ at $w_{j}$ and $Q=\left(w_{l}, e_{1}^{\prime}, v_{1}, \ldots, v_{q-1}, e_{q}^{\prime}, v_{q}\right)$ at $w_{l}$, where $2 \leq j<l \leq k$ and $p, q \geq 1$. Then $T \cong H_{w_{j}, w_{l}}(p, q)$, where $H=T\left[V(T) \backslash\left(V(P \cup Q) \backslash\left\{w_{j}, w_{l}\right\}\right)\right]$. Assume that $p \geq$ $q$. Note that $d_{H}\left(w_{j}\right)=d_{H}\left(w_{l}\right)=1$ and $T^{\prime}=H_{w_{j}, w_{l}}(p+1, q-1)$ is a $k$-uniform hypertree with maximum degree $\Delta$. By Corollary 3.1, we have $\rho(T)<\rho\left(T^{\prime}\right)$, a contradiction. Thus $T\left[V\left(T_{i}\right) \cup\{v\}\right]$ is a pendant path at $v$ for $2 \leq i \leq d_{T}(v)$ for $k \geq 2$. Let $l_{i}$ be the length of the pendant path $T\left[V\left(T_{i}\right) \cup\{v\}\right]$ at $v$, where $2 \leq i \leq d_{T}(v)$ and $l_{i} \geq 1$. Then $T \cong G_{v}\left(l_{2}, l_{3}\right)$, where $G=T\left[V(T) \backslash V\left(T_{2} \cup T_{3}\right)\right]$. Assume that $l_{2} \geq l_{3}$. Note that $T^{\prime \prime}=G_{v}\left(l_{2}+1, l_{3}-1\right)$ is a $k$-uniform hypertree with maximum degree $\Delta$. By Theorem 3.1, $\rho(T)<\rho\left(T^{\prime \prime}\right)$, a contradiction. Thus $u$ is the unique vertex of degree at least 3 in $T$.

Let $G_{1}, \ldots, G_{\Delta}$ be the vertex disjoint sub-hypergraphs of $T-u$ such that $T\left[V\left(G_{i}\right) \cup\{u\}\right]$ is a connected $k$-uniform hypergraph for $1 \leq i \leq \Delta$. By similar argument as above, $T\left[V\left(G_{i}\right) \cup\{u\}\right]$ is a pendant path at $u$ for $1 \leq i \leq \Delta$. Let $l_{i}$ be the length of the pendant path $T\left[V\left(G_{i}\right) \cup\{u\}\right]$ at $u$ for $1 \leq i \leq \Delta$. Suppose that there are at least two pendant paths of length at least two at $u$, say $T\left[V\left(G_{i}\right) \cup\{u\}\right]$ and $T\left[V\left(G_{j}\right) \cup\{u\}\right]$ are such two paths, where $1 \leq i<j \leq \Delta$. Then $T \cong H_{u}\left(l_{i}, l_{j}\right)$, where $H=T\left[V(T) \backslash V\left(G_{i} \cup G_{j}\right)\right]$. Assume that $l_{i} \geq l_{j}$. Note that $T^{*}=H_{u}\left(l_{i}+1, l_{j}-1\right)$ is also a $k$-uniform hypertree with maximum degree $\Delta$. By Theorem 3.1, $\rho(T)<\rho\left(T^{*}\right)$, a contradiction. Thus there is at most one pendant path of length at least 1 , implying that $T \cong B_{n, k}^{\Delta}$.

Case 2. $\Delta=2$.

It is trivial if $k=2$. Suppose that $k \geq 3$ and $T \not B_{n, k}^{2}$. Then there is an edge in $T$ with at least three vertices of degree 2. Choose such an edge $e=\left\{w_{1}, \ldots, w_{k}\right\}$ in $E(T)$ such that $d_{T}\left(u, w_{1}\right)$ is as large as possible, where $d_{T}\left(u, w_{1}\right)=d_{T}\left(u, w_{j}\right)-1$ for $2 \leq j \leq k$. Then there are two pendant paths at different vertices of $e$, say $P=\left(w_{j}, e_{1}, u_{1}, \ldots, u_{p-1}, e_{p}, u_{p}\right)$ at $w_{j}$ and $Q=\left(w_{l}, e_{1}^{\prime}, v_{1}, \ldots, v_{q-1}, e_{q}^{\prime}, v_{q}\right)$ at $w_{l}$, where $2 \leq j<l \leq k$ and $p, q \geq 1$. Then $T \cong H_{w_{j}, w_{l}}(p, q)$, where $H=T\left[V(T) \backslash\left(V(P \cup Q) \backslash\left\{w_{j}, w_{l}\right\}\right)\right]$. Assume that $p \geq q$. Note that $d_{H}\left(w_{j}\right)=d_{H}\left(w_{l}\right)=1$ and $T^{\prime}=H_{w_{j}, w_{l}}(p+1, q-1)$ is a $k$-uniform hypertree with maximum degree 2. By Corollary [3.1, we have $\rho(T)<\rho\left(T^{\prime}\right)$, a contradiction. Thus there are at most two vertices of degree two in each edge, implying that $T \cong B_{n, k}^{2}$.

Combining Cases 1 and 2, we complete the proof.

Theorem 4.2. Let $T$ be a k-uniform hypertree on $n$ vertices, where $\frac{n-1}{k-1} \geq 1$. Then $\rho(T) \leq \rho\left(P_{n, k}\right)$ with equality if and only if $T \cong P_{n, k}$.

Proof. It is trivial if $\frac{n-1}{k-1}=1,2$. Suppose that $\frac{n-1}{k-1} \geq 3$. Let $T$ be a $k$-uniform hypertree of order $n$ with maximum distance spectral radius. Let $\Delta$ be the 
maximum degree of $T$. Then by Theorem 4.1, $T \cong B_{n, k}^{\Delta}$. Suppose that $\Delta \geq 3$. By Theorem 3.1, we have $\rho\left(B_{n, k}^{\Delta}\right)<\rho\left(B_{n, k}^{\Delta-1}\right)$, a contradiction. Then $\Delta=2$, and thus $T \cong B_{n, k}^{2} \cong P_{n, k}$.

For $k \geq 3$ and a loose path $P_{n-k+1, k}=\left(u_{0}, e_{1}, u_{1}, \ldots, e_{\frac{n-k}{k-1}}, u_{\frac{n-k}{k-1}}\right)$, where $\frac{n-1}{k-1} \geq 3$, let $F_{n, k}$ be the $k$-uniform hypertree obtained from $P_{n-k+1, k}$ by attaching a pendant edge at a vertex of degree one in $e_{2}$. If $\frac{n-1}{k-1}=3$, then $F_{n, k} \cong P_{n, k}$.

Lemma 4.1. Suppose that $\frac{n-1}{k-1} \geq 3$ and $k \geq 3$. Then $\rho\left(B_{n, k}^{3}\right)<\rho\left(F_{n, k}\right)$.

Proof. If $\frac{n-1}{k-1}=3$, then the result follows from Theorem 4.2.

Suppose that $\frac{n-1}{k-1} \geq 4$. Let $T=F_{n, k}$. Let $v \in e_{2}$ such that $d_{T}(v)=1$, and let $e$ be the pendant edge at $v$. Let $T^{\prime}$ be the hypergraph obtained from $T$ by moving $e$ from $v$ to $u_{1}$. Obviously, $T^{\prime} \cong B_{n, k}^{3}$.

As we pass from $T$ to $T^{\prime}$, the distance between a vertex of $e \backslash\{v\}$ and a vertex of $e_{1}$ is decreased by 1 , the distance between a vertex of $e \backslash\{v\}$ and $v$ is increased by 1 , and the distance between any other vertex pair remained unchanged. Let $x=x\left(T^{\prime}\right)$. Then

$$
\begin{aligned}
\frac{1}{2}\left(\rho(T)-\rho\left(T^{\prime}\right)\right) x_{v} & \geq \frac{1}{2} x^{\top}\left(D(T)-D\left(T^{\prime}\right)\right) x \\
& =\left(\sigma_{T^{\prime}}(e)-x_{v}\right)\left(\sigma_{T^{\prime}}\left(e_{1}\right)-x_{v}\right) .
\end{aligned}
$$

From the eigenequations of $T$ at $u_{0}, u_{1}$ and $v$, we have

$$
\begin{aligned}
\rho\left(T^{\prime}\right) x_{u_{0}} & =x_{u_{1}}+2 x_{v}+\sum_{i=2}^{k-1} x_{w_{i}}+\sum_{w \in V(T) \backslash\left(e_{1} \cup\{v\}\right)} d_{T^{\prime}}\left(u_{0}, w\right) x_{w}, \\
\rho\left(T^{\prime}\right) x_{u_{1}} & =x_{u_{0}}+x_{v}+\sum_{i=2}^{k-1} x_{w_{i}}+\sum_{w \in V(T) \backslash\left(e_{1} \cup\{v\}\right)} d_{T^{\prime}}\left(u_{1}, w\right) x_{w}, \\
\rho\left(T^{\prime}\right) x_{v} & =2 x_{u_{0}}+x_{u_{1}}+\sum_{i=2}^{k-1} 2 x_{w_{i}}+\sum_{w \in V(T) \backslash\left(e_{1} \cup\{v\}\right)} d_{T^{\prime}}(v, w) x_{w} .
\end{aligned}
$$

Note that for $w \in V(T) \backslash\left(e_{1} \cup\{v\}\right), d_{T^{\prime}}\left(u_{0}, w\right)+d_{T^{\prime}}\left(u_{1}, w\right)-d_{T^{\prime}}(v, w) \geq 0$. Then $\left(\rho\left(T^{\prime}\right)+1\right)\left(x_{u_{0}}+x_{u_{1}}-x_{v}\right) \geq x_{u_{1}}+2 x_{v}>0$, and thus $\sigma_{T^{\prime}}\left(e_{1}\right)-x_{v}>$ $x_{u_{0}}+x_{u_{1}}-x_{v}>0$. It follows that $\rho\left(T^{\prime}\right)<\rho(T)$, as desired.

Let $T$ be a $k$-uniform hypertree of order $n$, where $T ¥ P_{n, k}$. Then $\frac{n-1}{k-1} \geq 3$, and if $\frac{n-1}{k-1}=3$, then $T \cong S_{n, k}$.

Let $F_{n, 2}=B_{n, 2}^{3}$.

Theorem 4.3. Let $T$ be a k-uniform hypertree of order $n$, where $T ¥ P_{n, k}$ and $\frac{n-1}{k-1} \geq 4$. Then $\rho(T) \leq \rho\left(F_{n, k}\right)$ with equality if and only if $T \cong F_{n, k}$. 
Proof. Let $T$ be a $k$-uniform hypertree of order $n$ nonisomorphic to $P_{n, k}$ with maximum distance spectral radius.

Let $\Delta$ be the maximum degree of $T$. Then $\Delta \geq 3$ if $k=2$ and $\Delta \geq 2$ if $k \geq 3$.

Suppose that $\Delta \geq 3$. Then by Theorem 4.1, $T \cong B_{n, k}^{\Delta}$. Suppose that $\Delta \geq 4$. Note that $B_{n, k}^{\Delta-1} \nsucceq P_{n, k}$. By Theorem 3.1, we have $\rho\left(B_{n, k}^{\Delta}\right)<\rho\left(B_{n, k}^{\Delta-1}\right)$, a contradiction. Thus $\Delta=3$. It follows that $T \cong B_{n, k}^{3}$. The result for $k=2$ follows.

Suppose in the following that $k \geq 3$.

Suppose that $\Delta=2$. Since $T ¥ P_{n, k}$, there is at least one edge with at least three vertices of degree 2. Suppose that there are at least two such edges. Let $u$ be a vertex of degree one in $T$. Choose an edge $e=\left\{w_{1}, \ldots, w_{k}\right\}$ in $E(T)$ with at least 3 vertices of degree two such that $d_{T}\left(u, w_{1}\right)$ is as large as possible, where $d_{T}\left(u, w_{1}\right)=d_{T}\left(u, w_{i}\right)-1$ for $2 \leq i \leq k$. Then there are at least two pendant paths at different vertices of $e$, say $P=\left(w_{i}, e_{1}, u_{1}, \ldots, u_{p-1}, e_{p}, u_{p}\right)$ at $w_{i}$ and $Q=\left(w_{j}, e_{1}^{\prime}, v_{1}, \ldots, v_{q-1}, e_{q}^{\prime}, v_{q}\right)$ at $w_{j}$, where $1 \leq i<j \leq k$ and $p, q \geq 1$. Then $T \cong H_{w_{i}, w_{j}}(p, q)$, where $H=T\left[V(T) \backslash\left(V(P \cup Q) \backslash\left\{w_{i}, w_{j}\right\}\right)\right]$. Assume that $p \geq q$. Note that $T^{\prime}=H_{w_{i}, w_{j}}(p+1, q-1)$ is a $k$-uniform hypertree that is not isomorphic to $P_{n, k}$. By Corollary 3.1. we have $\rho(T)<\rho\left(T^{\prime}\right)$, a contradiction. Thus there is exactly one edge $e$ with at least three vertex of degree 2.

We claim that there are exactly three vertices of degree two in $e$. Otherwise, $k \geq 4$ and there are four vertices $w_{1}, w_{2}, w_{3}$ and $w_{4}$ of degree two in $e$. Let $Q_{i}$ be the pendant path of length $l_{i}$ at $w_{i}$, where $l_{i} \geq 1$ for $i=1,2,3,4$. Assume that $l_{1} \geq l_{2}$. Let $G=T\left[V(T) \backslash\left(V\left(Q_{1} \cup Q_{2}\right) \backslash\left\{w_{1}, w_{2}\right\}\right)\right]$. Then $T \cong G_{w_{1}, w_{2}}\left(l_{1}, l_{2}\right)$, Note that $T^{\prime \prime}=G_{w_{1}, w_{2}}\left(l_{1}+1, l_{2}-1\right)$ is a $k$-uniform hypertree that is not isomorphic to $P_{n, k}$. By Corollary 3.1, $\rho(T)<\rho\left(T^{\prime \prime}\right)$, a contradiction. Thus there are exactly three vertices of degree two in $e$, say $w_{1}, w_{2}$, and $w_{3}$.

Let $Q_{i}$ be the pendant path at $w_{i}$ with length $l_{i}$, where $i=1,2,3$ and $l_{i} \geq 1$. Assume that $l_{1} \geq l_{2} \geq 2$. Let $G=T\left[V(T) \backslash\left(V\left(Q_{1} \cup Q_{2}\right) \backslash\left\{w_{1}, w_{2}\right\}\right)\right]$. Then $T \cong G_{w_{1}, w_{2}}\left(l_{1}, l_{2}\right)$. Note that $T^{*}=G_{w_{1}, w_{2}}\left(l_{1}+1, l_{2}-1\right)$ is a $k$-uniform hypertree that is not isomorphic to $P_{n, k}$. By Corollary 3.1, $\rho(T)<\rho\left(T^{*}\right)$, a contradiction. Thus there are at least two of $Q_{1}, Q_{2}$ and $Q_{3}$ with length 1 . Thus $T \cong F_{n, k}$.

By Lemma 4.1, $\rho\left(B_{n, k}^{3}\right)<\rho\left(F_{n, k}\right)$. Thus $T \cong F_{n, k}$.

Theorem 4.4. Let $T$ be a $k$-uniform hypertree on $n$ vertices, where $\frac{n-1}{k-1} \geq 1$. Then $\rho(T) \geq \rho\left(S_{n, k}\right)$ with equality if and only if $T \cong S_{n, k}$.

Proof. It is trivial if $\frac{n-1}{k-1} \leq 2$. Suppose that $\frac{n-1}{k-1} \geq 3$. Let $T$ be a $k$-uniform hypertree of order $n$ with minimum distance spectral radius.

Let $d$ be the diameter of $T$. Obviously, $d \geq 2$. Suppose that $d \geq 3$. Let $P=\left(v_{0}, e_{1}, v_{1}, \ldots, v_{d-1}, e_{d}, v_{d}\right)$ be a diametral path of $T$. Let $e_{d-1}=$ $\left\{w_{1}, w_{2}, \ldots, w_{k}\right\}$, where $w_{k-1}=v_{d-1}$ and $w_{k}=v_{d-2}$. For $1 \leq i \leq k-1$, let $t_{i}$ be the number of pendant edges at $w_{i}$ outside $P$, where $t_{k-1} \geq 1$. For 
$1 \leq i \leq k-1$ with $t_{i} \geq 1$, let $e_{i}^{1}, \ldots, e_{i}^{t_{i}}$ be the $t_{i}$ pendant edges at $w_{i}$ outside $P$, where $e_{w_{k-1}}^{1}=e_{s}$. Then $T \cong G_{e_{d-1}, 0}\left(t_{1}, \ldots, t_{k-1}\right)$, where $G=$ $T\left[V(T) \backslash E_{1}\right]$ and $E_{1}=\cup_{\substack{1 \leq i \leq k-1 \\ 1 \leq j \leq t_{i}}}\left(e_{i}^{j} \backslash\left\{w_{i}\right\}\right)$. Let $T^{\prime}$ be the hypergraph obtained from $G_{e_{d-1}, 0}\left(t_{1}, \ldots, t_{k-1}\right)$ by moving edges $e_{i}^{1}, \ldots, e_{i}^{t_{i}}$ from $w_{i}$ to $w_{k}$ for all $i$ with $1 \leq i \leq k-1$ and $t_{i} \geq 1$. Then $T^{\prime} \cong G_{e_{d-1}, k-1}\left(t_{1}, \ldots, t_{k}\right)$. By Corollary 3.2. $\rho(T)>\rho\left(T^{\prime}\right)$, a contradiction. Thus $d=2$, implying that $T \cong S_{n, k}$.

An automorphism of a hypergraph $G$ is a bijection on $V(G)$ which induces a bijection on $E(G)$.

Lemma 4.2. Let $G$ be a connected $k$-uniform hypergraph with $\eta$ being an automorphism of $G$. Let $x=x(G)$. Then $\eta(u)=v$ implies that $x_{u}=x_{v}$.

Proof. Let $P$ be the permutation matrix that corresponds to the automorphism $\eta$ of $G$. Then $D(G)=P^{\top} D(G) P$. Since $\rho(G) x=D(G) x$, we have $\rho(G)=x^{\top} D x=(P x)^{\top} D(G)(P x)$. Obviously, $P x$ is a positive unit vector. Thus $P x=x$, from which the result follows.

For $\frac{n-1}{k-1} \geq 3$ and $1 \leq a \leq\left\lfloor\frac{n-k}{2(k-1)}\right\rfloor$, let $D_{n, a}$ be the $k$-uniform hypertree obtained from vertex-disjoint $S_{a(k-1)+1, k}$ with center $u$ and $S_{n-k-a(k-1)+1, k}$ with center $v$ by adding $k-2$ new vertices $w_{1}, \ldots, w_{k-2}$ and an edge $\left\{u, v, w_{1}, \ldots, w_{k-2}\right\}$.

Lemma 4.3. For $2 \leq a \leq\left\lfloor\frac{n-k}{2(k-1)}\right\rfloor, \rho\left(D_{n, a}\right)>\rho\left(D_{n, a-1}\right)$.

Proof. Let $b=\frac{n-k}{k-1}-a$. Let $u$ and $v$ be the vertices of $D_{n, a}$ with degree $a$ and $b$, respectively. Let $E\left(D_{n, a}\right)=\left\{e_{i}: 1 \leq i \leq a+b+1\right\}$ and $e_{i}=\left\{w_{i}^{1}, \ldots, w_{i}^{k}\right\}$ for $1 \leq i \leq a+b+1$, where $w_{i}^{k}=u$ if $1 \leq i \leq a, w_{i}^{k}=v$ for $a+1 \leq i \leq a+b$, and $w_{i}^{1}=u$ and $w_{i}^{k}=v$ for $i=a+b+1$. Let $x=x\left(D_{n, a}\right)$. By Lemma 4.2, $x_{w_{i}^{1}}=x_{w_{i}^{j}}$ and $x_{w_{1}^{1}}=\cdots=x_{w_{a}^{1}}$ for $1 \leq i \leq a$ and $1 \leq j \leq k-1, x_{w_{i}^{1}}=x_{w_{i}^{j}}$ and $x_{w_{a+1}^{1}}=\cdots=x_{w_{a+b}^{1}}$ for $a+1 \leq i \leq a+b$ and $1 \leq j \leq k-1$, and $x_{w_{i}^{2}}=\cdots=x_{w_{i}^{k-1}}$ for $i=a+b+1$. Let $\alpha=x_{w_{1}^{1}}, \beta=x_{w_{a+1}^{1}}$, and $\gamma=x_{w_{a+b+1}^{2}}$. From the eigenequations of $D_{n, a}$ at $w_{1}^{1}, w_{a+1}^{1}, w_{a+b+1}^{1}, u$ and $v$, we have

$$
\begin{aligned}
\rho\left(D_{n, a}\right) \alpha & =(2(k-1) a-k) \alpha+3(k-1) b \beta+2(k-2) \gamma+x_{u}+2 x_{v}, \\
\rho\left(D_{n, a}\right) \beta & =3(k-1) a \alpha+(2(k-1) b-k) \beta+2(k-2) \gamma+2 x_{u}+x_{v}, \\
\rho\left(D_{n, a}\right) \gamma & =2(k-1) a \alpha+2(k-1) b \beta+(k-3) \gamma+x_{u}+x_{v}, \\
\rho\left(D_{n, a}\right) x_{u} & =(k-1) a \alpha+2(k-1) b \beta+(k-2) \gamma+x_{v}, \\
\rho\left(D_{n, a}\right) x_{v} & =2(k-1) a \alpha+(k-1) b \beta+(k-2) \gamma+x_{u} .
\end{aligned}
$$

We view these equations as a homogeneous linear system in the five variables $\alpha, \beta, \gamma, x_{u}$, and $x_{v}$. Since it has a nontrivial solution, we have

$$
\operatorname{det}\left(\begin{array}{ccccc}
2(k-1) a-k-\rho & 3(k-1) b & 2(k-2) & 1 & 2 \\
3(k-1) a & 2(k-1) b-k-\rho & 2(k-2) & 2 & 1 \\
2(k-1) a & 2(k-1) b & k-3-\rho & 1 & 1 \\
(k-1) a & 2(k-1) b & k-2 & -\rho & 1, \\
2(k-1) a & (k-1) b & k-2 & 1 & -\rho
\end{array}\right)=0
$$


where $\rho=\rho\left(D_{n, a}\right)$. Thus $\rho\left(D_{n, a}\right)$ is the largest root of the equation $g_{a}(t)=0$, where

$$
\begin{aligned}
g_{a}(t)= & -t^{5}+t^{4}(2 a k+2 b k-k-2 a-2 b-3) \\
& +t^{3}\left(k^{2}+4 a k^{2}+4 b k^{2}+5 a b k^{2}-10 a b k\right. \\
& -a k-b k-4 k+5 a b-3 a-3 b-3) \\
& +t^{2}\left(k^{3}+3 a b k^{3}+2 a k^{3}+2 b k^{3}+k^{2}-3 a b k^{2}\right. \\
& \left.+4 a k^{2}+4 b k^{2}-3 a b k-5 a k-5 b k-5 k+3 a b-a-b-1\right) \\
& +t\left(2 k^{3}+2 a b k^{3}+3 a k^{3}+3 b k^{3}-k^{2}-4 a b k^{2}\right. \\
& \left.-a k^{2}-b k^{2}+2 a b k-2 a k-2 b k-2 k\right) \\
& +k^{3}+a k^{3}+b k^{3}-k^{2}-a k^{2}-b k^{2} .
\end{aligned}
$$

For $2 \leq a \leq\left\lfloor\frac{n-k}{2(k-1)}\right\rfloor$, it is easily seen that

$$
g_{a}(t)-g_{a-1}(t)=(b+1-a)(k-1)^{2} t\left[5 t^{2}+(3 k+3) t+2 k\right] \text {. }
$$

Let $\rho_{i}=\rho\left(D_{n, i}\right)$ for $1 \leq i \leq\left\lfloor\frac{n-k}{2(k-1)}\right\rfloor$. Then

$$
\begin{aligned}
g_{a}\left(\rho_{a-1}\right) & =g_{a}\left(\rho_{a-1}\right)-g_{a-1}\left(\rho_{a-1}\right) \\
& =(b+1-a)(k-1)^{2} \rho_{a-1}\left[5 \rho_{a-1}^{2}+(3 k+3) \rho_{a-1}+2 k\right] \\
& >0
\end{aligned}
$$

from which, together with the fact that $g_{a}(t)<0$ for $t>\rho_{a}$, we have $\rho_{a}>\rho_{a-1}$ for $2 \leq a \leq\left\lfloor\frac{n-k}{2(k-1)}\right\rfloor$.

Theorem 4.5. Let $T$ be a k-uniform hypertree of order $n$, where $T ¥ S_{n, k}$ and $\frac{n-1}{k-1} \geq 3$. Then $\rho(T) \geq \rho\left(D_{n, 1}\right)$ with equality if and only if $T \cong D_{n, 1}$.

Proof. It is trivial if $\frac{n-1}{k-1}=3$. Suppose that $\frac{n-1}{k-1} \geq 4$. Let $T$ be a $k$-uniform hypertree of order $n$ nonisomorphic to $S_{n, k}$ with minimum distance spectral radius.

Let $d$ be the diameter of $T$. Since $T ¥ S_{n, k}$, we have $d \geq 3$. Let $P=$ $\left(v_{0}, e_{1}, v_{1}, \ldots, v_{d-1}, e_{d}, v_{d}\right)$ be a diametral path of $T$. Let $e_{d-1}=\left\{w_{1}, \ldots, w_{k}\right\}$, where $w_{k-1}=v_{d-1}$ and $w_{k}=v_{d-2}$. For $1 \leq i \leq k-1$, let $t_{i}$ be the number of the pendant edges at $w_{i}$ outside $P$, where $t_{k-1} \geq 1$. For $1 \leq i \leq k-1$ with $t_{i} \geq 1$, let $e_{i}^{1}, \ldots, e_{i}^{t_{i}}$ be the $t_{i}$ pendant edges at $w_{i}$ outside $P$, where $e_{w_{k-1}}^{1}=e_{d}$. Then $T \cong G_{e_{d-1}, 0}\left(t_{1}, \ldots, t_{k-1}\right)$, where $G=T\left[V(T) \backslash E_{1}\right]$ and $E_{1}=\cup_{\substack{1 \leq i \leq k-1 \\ 1 \leq j \leq t_{i}}}\left(e_{i}^{j} \backslash\left\{w_{i}\right\}\right)$. Assume that $T=G_{e_{d-1}, 0}\left(t_{1}, \ldots, t_{k-1}\right)$.

Suppose that $d \geq 4$. Let $T^{\prime}$ be the hypergraph obtained from $T$ by moving edges $e_{i}^{1}, \ldots, e_{i}^{t_{i}}$ from $w_{i}$ to $w_{k}$ for all $i$ with $1 \leq i \leq k-1$ and $t_{i} \geq 1$. Then $T^{\prime} \cong G_{e_{s-1}, k-1}\left(t_{1}, \ldots, t_{k-1}\right) \not S_{n, k}$. By Corollary 3.2, $\rho(T)>\rho\left(T^{\prime}\right)$, a contradiction. Thus $d=3$, implying that $T=G_{e_{2}, 0}\left(t_{1}, \ldots, t_{k-1}\right)$.

Suppose that $k \geq 3$ and $t_{i} \geq 1$ for some $i$ with $1 \leq i \leq k-2$. Let $T^{\prime \prime}$ be the hypergraph obtained from $T$ by moving edges $e_{i}^{1}, \ldots, e_{i}^{t_{i}}$ from $w_{i}$ to $w_{k}$ 
for all $i$ with $1 \leq i \leq k-2$ and $t_{i} \geq 1$. Then $T^{\prime \prime} \cong G_{e_{2}, k-2}\left(t_{1}, \ldots, t_{k-1}\right) \nsucceq$ $S_{n, k}$. By Corollary 3.2, $\rho(T)>\rho\left(T^{\prime \prime}\right)$, a contradiction. Thus $t_{i}=0$ for each $1 \leq i \leq k-2$. It follows that $T=G_{e_{2}, 0}\left(0, \ldots, 0, t_{k-1}\right) \cong D_{n, a}$, where $a=\min \left\{t_{k-1}, \frac{n-k}{k-1}-t_{k-1}\right\}$ for $k \geq 3$. Obviously, this is also true for $k=2$. By Lemma 4.3, we have $T \cong D_{n, 1}$

\section{Concluding remarks}

We propose three types of graft transformations for a $k$-uniform hypergraph, and study the effect of them to increase or decrease the distance spectral radius. We show that $P_{n, k}$ and $S_{n, k}$ are the unique $k$-uniform hypertrees with maximum and minimum distance spectral radius, respectively, and we also determine the unique $k$-uniform hypertrees with second maximum and second minimum distance spectral radius, respectively. Besides, we determine the unique hypertree with maximum distance spectral radius among $k$-uniform hypertrees with given maximum degree. Some theorems in this paper echo results on the distance spectral radius of ordinary graphs in the literature, see, e.g. [7, 9, 10, ?]. However, there are differences between $k$-uniform hypergraphs with $k \geq 3$ and ordinary graphs $(k=2)$. In Theorem 4.3 , we show that $F_{n, k}$ is the unique hypertree with second maximum distance spectral radius among $k$-uniform hypertrees of order $n$. It is easy to see that $F_{n, k}$ for $k \geq 3$ and $F_{n, 2}$ have quite different structure.

\section{References}

[1] M. Aouchiche, P. Hansen, Distance spectra of graphs: A survey, Linear Algebra Appl. 458 (2014) 301-386.

[2] C. Berge, Hypergraphs: Combinatorics of Finite Sets, North-Holland, Amsterdam, 1989.

[3] M. Edelberg, M.R. Garey, R.L. Graham, On the distance matrix of a tree, Discrete Math. 14 (1976) 23-39.

[4] R.L. Graham, L. Lovász, Distance matrix polynomials of trees, Adv. Math. 29 (1978) 60-88.

[5] R.L. Graham, H.O. Pollack, On the addressing problem for loop switching, Bell System Tech. J. 50 (1971) 2495-2519.

[6] M. Nath, S. Paul, On the distance spectral radius of trees, Linear Multilinear Algebra 61 (2013) 847-855. 
[7] S.N. Ruzieh, D.L. Powers, The distance spectrum of the path $P_{n}$ and the first distance eigenvector of connected graphs, Linear Multilinear Algebra 28 (1990) 75-81.

[8] S. Sivasubramanian, $q$-analogs of distance matrices of 3-hypertrees, Linear Algebra Appl. 431 (2009) 1234-1248.

[9] D. Stevanović, A. Ilić, Distance spectral radius of trees with fixed maximum degree, Electron. J. Linear Algebra 20 (2010) 168-179.

[10] Y. Wang, B. Zhou, On distance spectral radius of graphs, Linear Algebra Appl. 438 (2013) 3490-3503.

[11] R. Xing, B. Zhou, F. Dong, The effect of a graft transformation on distance spectral radius, Linear Algebra Appl. 457 (2014) 261-275. 